\title{
Etanercept and Adalimumab Treatment Patterns in Psoriatic Arthritis Patients Enrolled in a Commercial Health Plan
}

Benjamin Chastek · Kathleen M. Fox · Crystal Watson · Shravanthi R. Gandra

To view enhanced content go to www.advancesintherapy.com

Received: June 27, 2012 / Published online: August 16, 2012

(c) The Author(s) 2012. This article is published with open access at Springerlink.com

\section{ABSTRACT}

Introduction: Treatment patterns, including persistence, gaps in therapy, switching, and discontinuation, were examined in patients with psoriatic arthritis (PsA) who received the tumor necrosis factor (TNF)-blockers etanercept or adalimumab.

Methods: This retrospective study utilized administrative claims data from a United States commercial health plan. Adults (age 18-64 years) with PsA who started therapy with

B. Chastek $(\bowtie)$

OptumInsight, 12125 Technology Drive, Eden Prairie, MN 55344, USA

e-mail: Benjamin.Chastek@optum.com

\section{K. M. Fox}

Strategic Healthcare Solutions, LLC, P.O. Box 543,

Monkton, MD 21111, USA

C. Watson · S.R. Gandra

Amgen Inc., One Amgen Center Drive, Thousand Oaks, CA 91320, USA

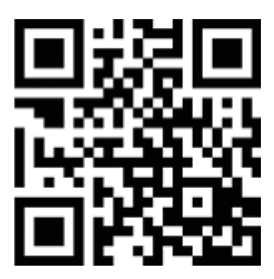

Enhanced content for Advances in Therapy articles is available on the journal web site: www.advancesintherapy.com etanercept or adalimumab as index therapy between January 1, 2006 and December 31, 2008 were included in the analysis. Patients were continuously enrolled in the health plan for at least 6 months before and at least 12 months after the start of index therapy. Initial TNF-blocker dose and rates of therapy persistence (continuous use of index medication without a gap of at least 60 days), therapy gaps, and discontinuation (gap in therapy of at least 60 days) were estimated. Those who discontinued were further classified as: (1) discontinued all biologic therapy, (2) restarted index medication, (3) switched to another biologic therapy, or (4) other.

Results: A total of 346 patients with PsA (202 etanercept, 144 adalimumab) were eligible. Most (90.6\% etanercept; $88.9 \%$ adalimumab) started index therapy at the labeled dose. Persistence with index therapy for 12 months was observed in $50 \%$ of patients on etanercept and $45 \%$ of patients on adalimumab $(P=0.37)$. Patients on etanercept had a longer duration of persistence (434 vs. 353 days; $P=0.02$ ), more pauses of at least 7 days ( 4.7 vs. $3.5 ; P=0.004)$, and a longer mean pause length (48.6 vs. 29.3 days; $P=0.01)$ than patients on adalimumab. Of patients who discontinued $(24.8 \%$ etanercept; 
$35.1 \%$ adalimumab), $46.4 \%$ and $41.5 \%$ restarted etanercept and adalimumab, respectively; $24.8 \%$ and $35.1 \%$ discontinued all TNF-blockers; $20.0 \%$ and $19.2 \%$ switched to another biologic; and $8.8 \%$ and $4.3 \%$ had other therapy changes. Conclusions: Approximately half of PsA patients were persistent on their index TNF-blocker for 12 months. Pauses in therapy and therapy discontinuation were common, but more than $40 \%$ of patients restarted their index TNFblocker after discontinuation.

Keywords: Adalimumab; Etanercept; Discontinuation; Persistence; Psoriatic arthritis; Switch; Treatment patterns

\section{INTRODUCTION}

Psoriatic arthritis (PsA) is a chronic, immunemediated, inflammatory disease with a wide global variation in prevalence, ranging up to 420 cases per 100,000 people [1]. Joint swelling and pain associated with PsA have a substantial negative impact on health-related quality of life [2,3]. Patients with PsA experience continuing disability, resulting in high rates of unemployment and work disability [4,5]. PsA is associated with significant socioeconomic and healthcare costs, which increase with disease severity and age [6-8].

Tumor necrosis factor (TNF)-blockers are effective for the treatment of many immunemediated inflammatory diseases, including PsA [9]. Etanercept (Immunex Corporation, Thousand Oaks, CA, USA) and adalimumab (Abbott Laboratories, North Chicago, IL, USA) are the most commonly prescribed TNFblockers approved for the treatment of PsA, and have been shown to reduce the signs and symptoms, inhibit the progression of structural damage from active arthritis, and improve the physical function of patients with PsA [10-13].
According to the prescribing information for PsA, etanercept should be administered at $50 \mathrm{mg}$ weekly and adalimumab should be administered at $40 \mathrm{mg}$ every other week (EOW) $[12,13]$.

Few studies have examined TNF-blocker treatment patterns in patients with PsA, and, to the authors' knowledge, no studies have examined treatment gaps, restarts, and switching in this patient population. A better understanding of treatment patterns would be useful to inform clinical practice decisions to ensure full treatment benefits and understand reasons for noncompliance. Saad et al. observed that $75.5 \%$ of patients with PsA in the British Society for Rheumatology Biologics Register (BSRBR) were persistent on their initial TNFblocker for 12 months [14]. The objective of this study was to describe real-world treatment patterns of etanercept and adalimumab in terms of therapy persistence, pauses in therapy, therapy discontinuation, therapy restarts, and therapy switches among PsA patients in a managed care population.

\section{METHODS}

\section{Study Design}

This retrospective cohort study used administrative claims data from a large, national United States (US) commercial health plan affiliated with OptumInsight ${ }^{\mathrm{TM}}$. The Life Sciences Research Database (formerly the Ingenix database) comprises fully adjudicated medical and pharmacy claims for over 27 million adults across the US.

\section{Patients}

Patients with a diagnosis of PsA who started therapy with etanercept or adalimumab as index therapy between January 1, 2006 and December 31, 2008 were included in the analysis. To be eligible, 
patients were adults (age 18-64 years) at the time of therapy initiation, were continuously enrolled in the health plan for at least 6 months before and at least 12 months after initiation of therapy, and had at least one claim with a diagnosis of PsA (ICD-9 696.0) from 6 months before through 30 days after therapy initiation. Patients were excluded from the study if they had a diagnosis for which TNF-blockers are often used, including rheumatoid arthritis (ICD-9 714.2), juvenile idiopathic arthritis (ICD-9 714.3), ankylosing spondylitis (ICD-9 720.0), Crohn's disease (ICD-9 555.2), ulcerative colitis (ICD-9 556.8), or psoriasis (ICD-9 696.1) during the 6 months prior to therapy initiation, through disenrollment or end of study. Patients with cancer (ICD-9 140.x-239.x) or human immunodeficiency virus infection (ICD-9 042.x) were also excluded. Diagnosis codes in all positions on the medical claim (i.e., primary and secondary diagnoses) were used throughout the inclusion and exclusion periods. Patients could not have had etanercept or adalimumab administered in a physician's office (evidence that the patient did not self-administer medications) or have a claim for other biologics of interest (abatacept, adalimumab, alefacept, anakinra, efalizumab, etanercept, golimumab, infliximab, rituximab, ustekinumab) during the 6 months prior to therapy initiation.

\section{Study Measures}

The initial TNF-blocker dose was defined as the average weekly dose of the initial prescription fill based on the quantity, drug strength, and number of days' supply. Patients were categorized according to their initial weekly dose of etanercept (50 mg weekly, $<50 \mathrm{mg}$ weekly, or other) or adalimumab (40 mg weekly, $40 \mathrm{mg}$ EOW, or other). Gaps in therapy were defined as the time between the run-out of a fill until the date of the next prescription refill. Gaps in therapy of at least 7 days (pauses in therapy) were identified. Pauses in therapy were not identified following the last prescription fill. Therapy persistence was defined as continuous use of index medication without gaps in therapy of at least 60 days. Patients with a gap in index therapy of at least 60 days were classified as discontinuing their index TNF-blocker and were further classified into one of four mutually exclusive groups: (1) discontinued biologic therapy (did not receive any other biologic therapy after the gap of at least 60 days), (2) restarted index therapy (after the gap of at least 60 days), (3) switched (started another biologic agent after the index biologic), and (4) other (e.g., switched to another biologic and then switched back to index medication).

\section{Statistical Analysis}

Statistical tests of significance for differences between etanercept and adalimumab used the chi-squared test for categorical variables; $t$ tests and analysis of variance (ANOVA) were used for normally distributed continuous variables, and nonparametric Wilcoxon and Kruskal-Wallis tests were used for continuous variables not normally distributed.

\section{RESULTS}

\section{Patient Characteristics and Initial Dose}

Among 346 eligible patients with PsA, $202(58.4 \%)$ started on therapy with etanercept and $144(41.6 \%)$ started therapy with adalimumab. Demographic characteristics were similar between groups (Table 1). The majority of patients (54-56\%) were from the southern region of the US and most (74-79\%) received their care from a rheumatologist. 
The majority of patients started their therapy at the dose recommended by the Food and Drug Administration. The most common comorbidity in both groups was hypertension. Of patients starting therapy with etanercept, $3.0 \%$ started at less than $50 \mathrm{mg}$ weekly, 90.6\% started at $50 \mathrm{mg}$ weekly, and $6.4 \%$ started at $100 \mathrm{mg}$ weekly. Of patients starting therapy with adalimumab, $0.7 \%$ started at less than $40 \mathrm{mg}$ EOW, 88.9\% started at $40 \mathrm{mg}$ EOW, and $10.4 \%$ started at more than $40 \mathrm{mg}$ weekly.

\section{Therapy Persistence}

In the first year of TNF-blocker therapy, similar proportions of patients starting on etanercept (50\%) and patients starting on adalimumab (45\%) were persistent (no gaps in at least 60 days) on their index medication $(P=0.37)$. The mean duration of persistence was 434 days (standard deviation $[\mathrm{SD}]=347$; 95\% confidence interval $[\mathrm{CI}]=385-482$ ) for patients on etanercept and 353 days $(\mathrm{SD}=298$;

Table 1 Demographics and disease characteristics of 346 patients with psoriatic arthritis who started treatment with etanercept or adalimumab

\begin{tabular}{|c|c|c|}
\hline & $\begin{array}{l}\text { Etanercept } \\
(n=202)\end{array}$ & $\begin{array}{l}\text { Adalimumab } \\
\qquad(n=144)\end{array}$ \\
\hline Male sex $(\%)$ & 56.4 & 56.9 \\
\hline Age (years, mean $\pm S D)$ & $45.6 \pm 10.9$ & $45.0 \pm 10.3$ \\
\hline Range & $18-82$ & $18-76$ \\
\hline \multicolumn{3}{|l|}{ Geographic location in USA (\%) } \\
\hline Northeast & 10.9 & 13.9 \\
\hline Midwest & 26.2 & 21.5 \\
\hline South & 53.5 & 56.2 \\
\hline West & 9.4 & 8.3 \\
\hline \multicolumn{3}{|l|}{ Index provider (\%) } \\
\hline Primary care & 14.5 & 8.4 \\
\hline Dermatologist & 3.0 & 1.4 \\
\hline Rheumatologist & 74.5 & 78.9 \\
\hline Other/unknown & 8.0 & 11.3 \\
\hline \multicolumn{3}{|l|}{ Quan-Charlson comorbidity index (\%) } \\
\hline $0-1$ & 93.6 & 96.5 \\
\hline 2 & 4.5 & 2.1 \\
\hline 3 or more & 2.0 & 1.4 \\
\hline \multicolumn{3}{|l|}{ Select comorbidities (\%) } \\
\hline Hypertension & 22.8 & 22.9 \\
\hline Ischemic heart disease & 4.0 & 4.2 \\
\hline Venous disease & 3.5 & 3.5 \\
\hline Diabetes & 12.4 & 8.3 \\
\hline $\begin{array}{l}\text { Methotrexate use in } 6 \text { months prior to initiation of index } \\
\text { medication (\%) }\end{array}$ & 31.2 & 41.0 \\
\hline
\end{tabular}

SD standard deviation 
95\% CI $=304-402)$ for patients on adalimumab $(P=0.02)$.

\section{Pauses in Therapy}

Etanercept patients had a significantly greater mean number of pauses $(4.7 ; \mathrm{SD}=4.3 ; 95 \% \mathrm{CI}=$ 4.1-5.3) than did adalimumab patients (3.5; SD $=3.4 ; 95 \% \mathrm{CI}=3.0-4.1 ; P=0.004)$, and a longer mean pause length (48.6 days; $\mathrm{SD}=95.0 ; 95 \%$ $\mathrm{CI}=35.4-61.7)$ than adalimumab patients $(29.3$ days; $\mathrm{SD}=37.9 ; 95 \% \mathrm{CI}=23.0-35.5 ; P=0.01$ ).

\section{Treatment Patterns in Discontinued Therapy Patients}

Among PsA patients, 125 patients on etanercept (61.9\%) and 94 patients on adalimumab (65.3\%) had a gap in index therapy of at least

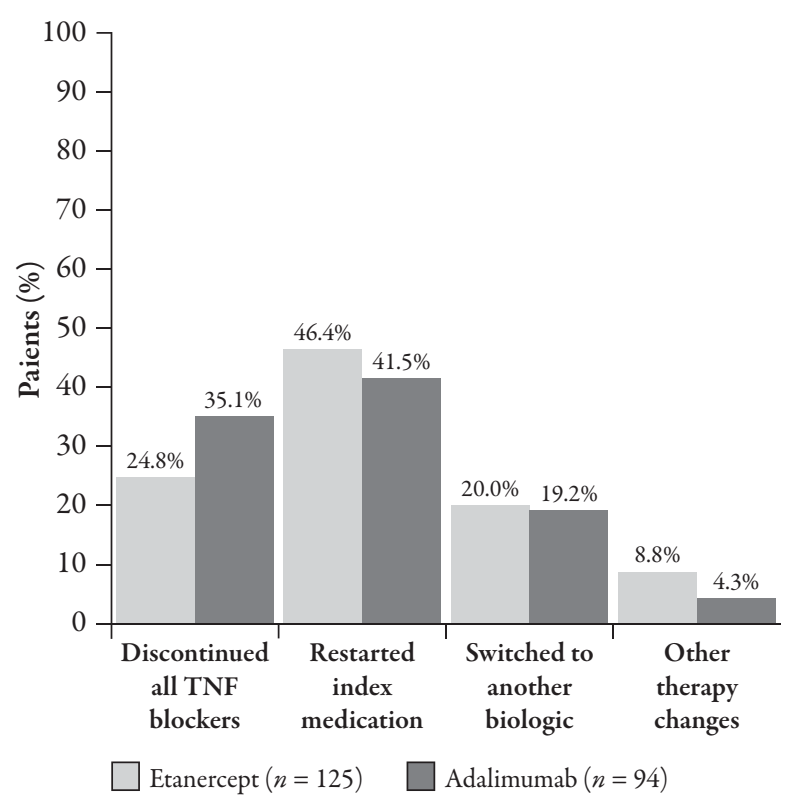

Fig. 1 Treatment patterns in patients with psoriatic arthritis, showing the proportion of patients with at least a 60-day gap in index therapy (219 out of a total of 346 patients in the study) who went on to discontinue all TNFblockers, restart index medication, or switch to another biologic, or had other changes in therapy. TNF tumor necrosis factor
60 days (i.e., they discontinued therapy). Among patients who discontinued therapy, rates of sustained discontinuation of the index medication without restart or switch were similar between patients who started on etanercept (24.8\%) and those who started on adalimumab (35.1\%; $P=0.10$ ) (Fig. 1). Similar percentages of patients restarted their index TNF-blocker after a gap in therapy of at least 60 days: $46.4 \%$ of patients on etanercept and $41.5 \%$ of patients on adalimumab $(P=0.47)$. Approximately $20 \%$ of patients who started on etanercept and $19 \%$ of patients who started on adalimumab switched to another biologic to treat their PsA after at least a 60 -day gap $(P=0.88)$. The remaining $4.3 \%$ of patients treated with etanercept and $8.8 \%$ of patients treated with adalimumab followed an alternative treatment pattern after their initial discontinuation, such as temporarily switching to a different biologic and then switching back to the index treatment.

\section{DISCUSSION}

The present study evaluated biologic treatment patterns among PsA patients; variables measured included persistence, pauses in therapy, restarts, switches, and discontinuation. Treatment patterns were similar for patients who started biologic therapy with etanercept and those who started therapy with adalimumab for the treatment of PsA in this US commercial health plan. For both etanercept and adalimumab, the majority of patients started at the recommended labeled dosing. Approximately 50\% of patients with PsA were persistent on their index TNFblocker for at least 1 year. Both patients receiving etanercept and those receiving adalimumab had an average of three to four pauses in therapy of at least 7 days. The percentage of patients with at least a 60-day gap was high (62-65\%), but of these patients more than $40 \%$ restarted 
their index TNF-blocker and an additional 20\% switched to another TNF-blocker.

In a study of 566 PsA patients enrolled in the BSRBR, $75.5 \%$ of patients were persistent on their index TNF-blocker at 12 months [14]. This rate is higher than the persistence rates observed in the present study's US population (50\% of patients on etanercept and $45 \%$ of patients on adalimumab). This difference may be attributable to the definition of persistence used in each study; gaps in therapy up to 3 months were allowed in the BSRBR study, to accommodate treatment of infections or for elective surgery, whereas patients in the present study were considered discontinued if they did not restart index medication after a 60-day gap [14]. The rates of discontinuation without switch or restart were similar between the BSRBR study (24.5\%) [14] and the present study $(24.8 \%$ of patients on etanercept and $35.1 \%$ of patients on adalimumab).

The findings of this study may be generalizable to PsA patients who receive etanercept or adalimumab in US-managed healthcare plans. Limitations of the study include those due to the nature of claims-based analyses, such as the ability of these data to accurately capture individual medical history and comorbidities that affect treatment decisions and reasons for discontinuation. The study population was restricted to those patients who had at least 18 months of continuous enrollment in the health plan ( 6 months before and a minimum of 12 months after therapy initiation) and started either etanercept or adalimumab therapy; this group of patients may have a different overall health status than the general population of PsA patients. This study focused on the biologic therapies etanercept and adalimumab and did not evaluate other systemic therapies or the potential effect of concomitant therapies, such as methotrexate, on treatment patterns. This study may not be generalizable to other PsA populations, such as the entire US population, or Medicare, underinsured, or uninsured patients.

The treatment patterns observed in this study showed that approximately half of the PsA population had a change in therapy within the first year of starting a biologic therapy, and many of these restarted or switched biologics. The reasons for switching are unknown and likely include adverse events, lack of efficacy, and nonclinical issues such as changes in formulary or copay burden. This study showed that patients receiving etanercept had a longer duration of persistency than those on adalimumab in this US commercial health plan, although the percentages of patients who were persistent on therapy did not vary significantly between the two groups. There were also more gaps in therapy with etanercept (4.7 for etanercept vs. 3.5 for adalimumab) and the gaps were longer (49 days for etanercept vs. 29 days for adalimumab). The additional length of therapy may in part be accounted for by the gaps and time off therapy. The reasons for the differences between adalimumab and etanercept could not be determined from the claims data used in this analysis.

\section{CONCLUSION}

In this retrospective analysis of real-world treatment patterns for etanercept and adalimumab in a US commercial health plan, treatment patterns were similar for patients receiving etanercept and adalimumab. Approximately half of PsA patients were persistent on their index medication for 12 months and pauses and gaps in therapy were common. Of patients who discontinued their index medication, more than 40\% restarted their index therapy. Further studies to investigate the reasons for and consequences of discontinuations, pauses, and gaps in therapy are warranted, as minimizing gaps in therapy would 
be expected to maximize the clinical benefits of TNF-blocker therapy in patients with PsA.

\section{ACKNOWLEDGMENTS}

The authors thank Julia R. Gage, PhD, whose work was funded by Amgen Inc., for assistance with writing the manuscript. Mr. Chastek is the guarantor for this article, and takes responsibility for the integrity of the work as a whole.

Funding Statement. This study was sponsored by Immunex, a wholly owned subsidiary of Amgen Inc. and by Wyeth, which was acquired by Pfizer Inc. in October 2009.

Conflict of Interest. B.C. is an employee of OptumInsight, which received funding from Amgen Inc. for this study. K.M.F. is a consultant for Amgen Inc. C.W. was an employee of Amgen Inc. at the time the work was performed and owns Amgen stock. S.R.G. is an employee and shareholder of Amgen Inc.

Open Access. This article is distributed under the terms of the Creative Commons Attribution Noncommercial License which permits any noncommercial use, distribution, and reproduction in any medium, provided the original author(s) and source are credited.

\section{REFERENCES}

1. Dhir V, Aggarwal A. Psoriatic arthritis: a critical review. Clin Rev Allergy Immunol. 2012. [Epub ahead of print].

2. Salaffi F, Carotti M, Gasparini S, Intorcia M, Grassi W. The health-related quality of life in rheumatoid arthritis, ankylosing spondylitis, and psoriatic arthritis: a comparison with a selected sample of healthy people. Health Qual Life Outcomes. 2009;7:25.

3. Strand V, Sharp V, Koenig AS, et al. Comparison of health-related quality of life in rheumatoid arthritis, psoriatic arthritis and psoriasis and effects of etanercept treatment. Ann Rheum Dis. 2012;71:1143-50.
4. Tillett W, de-Vries C and McHugh NJ. Work disability in psoriatic arthritis: a systematic review. Rheumatology (Oxford). 2012;51:275-83.

5. Verstappen SM, Watson KD, Lunt M, McGrother $\mathrm{K}$, Symmons DP, Hyrich KL. Working status in patients with rheumatoid arthritis, ankylosing spondylitis and psoriatic arthritis: results from the British Society for Rheumatology Biologics Register. Rheumatology (Oxford). 2010;49:1570-77.

6. Poole CD, Lebmeier M, Ara R, Rafia R, Currie CJ. Estimation of health care costs as a function of disease severity in people with psoriatic arthritis in the UK. Rheumatology (Oxford). 2010;49:1949-56.

7. Zhu TY, Tam LS, Leung YY, et al. Socioeconomic burden of psoriatic arthritis in Hong Kong: direct and indirect costs and the influence of disease pattern. J Rheumatol. 2010;37:1214-20.

8. Olivieri I, de Portu S, Salvarani C, et al. The psoriatic arthritis cost evaluation study: a cost-ofillness study on tumour necrosis factor inhibitors in psoriatic arthritis patients with inadequate response to conventional therapy. Rheumatology (Oxford). 2008;47:1664-70.

9. Keystone EC, Ware CF. Tumor necrosis factor and anti-tumor necrosis factor therapies. J Rheumatol Suppl. 2010;85:27-39.

10. Mease PJ, Kivitz AJ, Burch FX, et al. Etanercept treatment of psoriatic arthritis: safety, efficacy, and effect on disease progression. Arthritis Rheum. 2004;50:2264-72.

11. Mease PJ, Gladman DD, Ritchlin CT, et al. Adalimumab for the treatment of patients with moderately to severely active psoriatic arthritis: results of a double-blind, randomized, placebocontrolled trial. Arthritis Rheum. 2005;52:3279-89.

12. Enbrel $^{\circledR}$ (etanercept). Prescribing information, medication guide, and instructions for use. Available at: www.enbrel.com/prescribinginformation.jspx. Accessed Jul 262012.

13. Humira ${ }^{\circledR}$ (adalimumab). Full prescribing information. Available at: www.rxabbott.com/pdf/ humira.pdf. Accessed Jul 262012.

14. Saad AA, Ashcroft DM, Watson KD, Hyrich KL, Noyce PR, Symmons DP. Persistence with antitumour necrosis factor therapies in patients with psoriatic arthritis: observational study from the British Society of Rheumatology Biologics Register. Arthritis Res Ther. 2009;11:R52. 